\title{
Implementation of Comparative Probability by Normal States. Infinite Dimensional Case
}

\author{
Simba A. Mutangadura
}

Department of Physics, University of Zimbabwe, Box M.P. 167, Mt. Pleasant, Harare, Zimbabwe` and International Centre for Theoretical Physics, Trieste, Italy

\begin{abstract}
Let $\mathscr{H}$ be an infinite dimensional Hilbert space and $\mathscr{P}(\mathscr{H})$ the set of all (orthogonal) projections on $\mathscr{H}$. A comparative probability on $\mathscr{P}(\mathscr{H})$ is a linear preorder $\leqq$ on $\mathscr{P}(\mathscr{H})$ such that $\mathbf{O} \leqq P \leqq \mathrm{l}, \mathbf{l} \npreceq \mathbf{O}$ and such that if $P \perp R$, $Q \perp R$, then $P \leqq Q \Leftrightarrow P+R \leqq Q+R$ for all $\bar{P}, Q, R$ in $\mathscr{P}(\mathscr{H})$. We give a sufficient and necessary condition for $\leqq$ to be implemented in a canonical way by a normal state on $\mathscr{B}(\mathscr{H})$, the bounded linear operators on $\mathscr{H}$.
\end{abstract}

\section{Introduction and Notation}

Let $\mathscr{H}$ be a Hilbert space. $\mathscr{P}(\mathscr{H})$ denotes the set of all (orthogonal) projections on $\mathscr{H}$. If $E$ is a closed subspace of $\mathscr{H}$, and $\phi \in \mathscr{H}$ then $P_{E}$ and $P_{\phi}$ denote the corresponding projections. We drop the $E$ and $\phi$ if no reference to the subspaces is required. $\mathscr{P}_{1}(\mathscr{H})$ is the subset of all one dimensional projections and $\mathscr{P}_{\sigma}(\mathscr{H})$ is the subset of all those projections $P_{E}$ such that $E$ is a separable (finite or infinite dimensional) subspace of $\mathscr{H}$. Lower case Roman subscripts as in $P_{j}$ or $P_{\phi_{k}}$ will generally be used for indexing sequences and nets. $\mathbf{N}, \mathbf{R}$ and $\mathbf{C}$ denote the natural numbers, the reals and the complex numbers respectively. All vectors of $\mathscr{H}$ appearing may be assumed to be normalised. $P_{\mathscr{H}}$ is denoted by $\mathbf{l}_{\mathscr{H}}$ or just $\mathbf{l}$ of no confusion arises and the zero vector is denoted by $\mathbf{O}$. The orthogonal complement of $P$ (i.e. $1-P$ ) is denoted by $P^{\perp}$. If $P, Q \in \mathscr{P}(\mathscr{H})$ and $P \leqq Q^{\perp}$ then we write $P \perp Q$.

Definition 1.1. Let $\mathscr{H}$ be a Hilbert space. A preorder relation $\leqq$ on $\mathscr{P}(\mathscr{H})$ is called an elementary comparative probability (ECP) iff the following axioms are satisfied by all $P, Q, R \in \mathscr{P}(\mathscr{H})$ :

A1 $P \leqq Q$ or $Q \leqq P$,

A2 $P \leqq Q$ and $Q \leqq R \Rightarrow P \leqq R$,

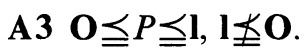

\footnotetext{
* Permanent address
} 
An $\mathrm{ECP} \leqq$ is called a comparative probability (CP) iff $\leqq$ satisfies :

A4 If $P \perp R, Q \perp R$, then $P \leqq Q \Leftrightarrow P+R \leqq Q+R \forall P, Q, R \in \mathscr{P}(\mathscr{H})$.

Recall that a (normalized) Gleason measure on $\mathscr{P}(\mathscr{H})$ is a mapping $\mu: \mathscr{P}(\mathscr{H}) \rightarrow[0,1]$ which is $\sigma$-orthoadditive and satisfies $\mu(\mathbf{l})=1$. If $\operatorname{dim} \mathscr{H} \geqq 3$, and $\mathscr{H}$ remains separable, then by Gleason's theorem [1], the set of all Gleason measures is exactly the set of all normal (i.e. $\sigma$-weakly continuous) states on $\mathscr{B}(\mathscr{H})$, the bounded linear operators on $\mathscr{H}$. If $\mathscr{H}$ is no longer separable then Gleason's theorem still holds providing the $\sigma$-orthoadditivity is strengthened to complete orthoadditivity or providing one assumes the generalized continuum hypothesis (see Kalmbach [2] Chapter 3) with only the $\sigma$-orthoadditivity.

It is clear that every normal state $\omega$ on $\mathscr{B}(\mathscr{H})$ induces a $\mathrm{CP} \leqq \omega$ on $\mathscr{P}(\mathscr{H})$ according to the prescription:

$$
P \leqq \omega \Leftrightarrow \omega(P) \leqq \omega(Q) .
$$

The purpose of this paper is to investigate the inverse problem. Specifically we wish to find the sufficient and necessary conditions for a CP on $\mathscr{P}(\mathscr{H})$, where $\mathscr{H}$ is a real or complex infinite dimensional Hilbert space, to be implemented by a normal state (=completely additive Gleason measure) in the sense of (1) above. The special and somewhat pathological case $\operatorname{dim} \mathscr{H}=2$ was resolved in [3], where the question of uniqueness of implementation was also investigated. It is worth noting that the case $\operatorname{dim} \mathscr{H}=2$ is extra special in that not all the axioms can be brought to bear on $\leqq$; in particular A4 has no effect and is replaced in [3] by:

$$
P \leqq Q \Leftrightarrow Q^{\perp} \leqq P^{\perp} \forall P, Q \in \mathscr{P}(\mathscr{H}) \text {. }
$$

We shall use the notation $P \cong Q$ to indicate $P \leqq Q$ and $Q \leqq P$; and $P \prec Q$ to denote $P \leqq Q$ and $Q \npreceq P$. If $E$ is a subspace of $\mathscr{H}$, the set $[P]_{E}$ is defined to be all $Q \in E$ such that $Q \cong P$ and $[\mathscr{P}(E)]$ to be the set of all $[Q]_{E}$ such that $Q$ is in $\mathscr{P}(E)$, the subscript $E$ being dropped if $E=\mathscr{H}$.

\section{Preliminaries}

The existence of states that are not normal shows that not every $\mathrm{CP}$ can be implemented by a normal state. In fact one can also show that for every dimension, finite or infinite, examples exist of CP's that cannot be implemented by states, normal or not. We need to impose some sort of "continuity" condition on $\leqq$ for it to be implementable by a normal state.

Definition 2.1. Let $\leqq$ be an ECP on $\mathscr{P}(\mathscr{H})$. We say that $\leqq$ is weakly continuous (or just continuous if no confusion arises) if whenever the net $P_{j}$ in $\mathscr{P}(\mathscr{H})$ converges weakly to $P$ and $P_{2} \leqq P_{j} \leqq P_{1} \forall j$, then $P_{2} \leqq P \leqq P_{1}$.

Let $\leqq$ be an ECP on $\mathscr{P}(\mathscr{H})$. Recall that the interval topology on $\mathscr{P}(\mathscr{H})$ induced by the linear preorder $\leqq$ is generated by a neighbourhood base consisting of $\leqq$ intervals of the form $(P, Q)$, where $P \prec Q,[\mathbf{O}, P)$ where $\mathbf{O} \prec P$, and $(P, \mathbf{l}]$ where $P \prec \mathbf{l}$. Continuity of $\leqq$ can be reformulated in terms of the strength of the $\leqq$ (interval) topology relative to that of the weak topology on $\mathscr{P}(\mathscr{H})$ (induced by the weak topology on $\mathscr{B}(\mathscr{H})$ ). Before showing this we first explain some notation: $P_{j} \stackrel{w}{\longrightarrow} P$ and $P_{j} \stackrel{\supseteqq}{\longrightarrow}$ imply convergence in the weak and in the $\leqq$ topologies respectively. 
Proposition 2.2. Let $\leqq$ be an $\mathrm{ECP}$ on $\mathscr{P}(\mathscr{H})$. Then $\leqq$ is continuous if and only if the topology on $\mathscr{P}(\mathscr{H})$ is weaker than the weak topology on $\mathscr{P}(\mathscr{H})$.

Proof. Let $\leqq$ be continuous. Suppose $P_{j}$ is a net in $\mathscr{P}(\mathscr{H})$ such that $P_{j} \stackrel{w}{\longrightarrow} P$, where $\mathbf{O} \prec P \prec \mathbf{l}$. Let $P^{\prime}$ and $P^{\prime \prime}$ be such that $P^{\prime} \prec P \prec P^{\prime \prime}$ so that $\left(P^{\prime}, P^{\prime \prime}\right)$ is a $\leqq$ neighbourhood of $P$. Then for some $j_{0}, P_{j} \in\left(P^{\prime}, P^{\prime \prime}\right) \forall j \geqq j_{0}$, lest we should find a subnet $P_{j_{k}}$ such that $\forall j_{k}, P_{j_{k}} \leqq P^{\prime}$ or $P^{\prime \prime} \leqq P_{j_{k}}$. This would, by continuity of $\leqq$ imply that $P \leqq P^{\prime}$ or $P^{\prime \prime} \leqq P$, a contradiction. Hence $P_{j} \leqq P$. Only trivial modification is required should $P \cong \mathbf{O}$ or $P \cong \mathbf{l}$. Thus the $\leqq$ topology is weaker than the weak topology on $\mathscr{P}(\mathscr{H})$.

Now let the $\leqq$ topology be weaker than the weak topology. Let $P_{j} \stackrel{w}{\longrightarrow} P$ and let $P^{\prime}, P^{\prime \prime}$ be such that $P^{\prime} \leqq P_{j} \leqq P^{\prime \prime} \forall j$. Suppose that $P^{\prime \prime} \prec P$, then there exist $R \in \mathscr{P}(\mathscr{H})$ such that $P \leqq R$ and such that $\left(P^{\prime \prime}, R\right]$ is a $\leqq$ neighbourhood, and hence by hypothesis, a weak neighbourhood of $P$. Thus there exists $j \in N$ such that $P_{j} \in(P, R]$ which implies $P \prec P_{j}$, a contradiction. Hence $P \leqq P^{\prime \prime}$. A similar argument establishes that $P^{\prime} \leqq P$ and the result follows.

Let $\mathscr{H}$ be an infinite dimensional Hilbert space. As is the case when dealing with normal states on $\mathscr{B}(\mathscr{H})$, separability of $\mathscr{H}$ is a natural condition when dealing with continuous CP's on $\mathscr{P}(\mathscr{H})$. This is obvious from the following:

Proposition 2.3. Let $\mathscr{H}$ be any (not necessarily separable) Hilbert space and $\leqq$ be a continuous $\mathrm{CP}$ on $\mathscr{P}(\mathscr{H})$.

i) Let $\mathscr{A} \subset \mathscr{P}_{1}(\mathscr{H})$ be any set of mutually orthogonal (one dimensional) projections such that $\forall P \in \mathscr{A}, \mathbf{O} \prec P$. Then $\mathscr{A}$ is at most a countably infinite set.

ii) If $E$ is a subspace of $\mathscr{H}$ such that $P \in \mathscr{P}_{1}(E) \Rightarrow P \cong \mathbf{O}$, then $P_{E} \cong \mathbf{O}$.

Proof. i) If $\mathscr{A}$ is a finite set there is nothing to prove; so assume that it is infinite. Now for any $P \in \mathscr{A}$ define $\mathscr{A}(P)$ to be $\{Q \in \mathscr{A}: P \leqq Q\}$. We note that $\mathscr{A}(P)$ is a finite set for each $P \in \mathscr{A}$, otherwise we can find in $\mathscr{A}(P)$ a sequence $Q_{j}$ such that $Q_{j} \stackrel{w}{\longrightarrow} \mathbf{O}$ and hence, by continuity of $\leqq, Q_{j} \leqq \mathbf{O}$. This contradicts the requirement that for all $j, \mathbf{O} \prec P \leqq Q_{j}$.

Now let $\left\{P_{j}: j \in \mathbf{N}\right\}$ be a countably infinite subset of $\mathscr{A}$. Clearly $P_{j} \supseteqq \mathbf{O}$ as $j \rightarrow \infty$. Hence we must have $\mathscr{A}=\bigcup_{j \in \mathrm{N}} \mathscr{A}\left(P_{j}\right)$ and the result follows as each $\mathscr{A}\left(P_{j}\right)$ is finite.

ii) The result is clear for $E$ separable, so assume that $E$ is not separable. Let $\mathscr{Z}$ be the set of all projections $P$ in $\mathscr{P}(E)$ such that $P \cong \mathbf{O}$. Then $\mathscr{Z}$ is a nonempty $\leqq$ closed subset of $\mathscr{P}(\mathscr{H})$. Let $P_{F_{j}}$ be an (operator theoretic) increasing net in $\mathscr{Z}$. Then $P_{F_{j}}$ is evidently a weakly convergent net which weakly and hence $\leqq$ converges to $P_{F}$, where $F$ is the closure of the linear span of $\cup_{j} F_{j}$. Thus, by Zorn's lemma, $\mathscr{Z}$ has an operator theoretic maximal projection $Q$, say. But we cannot have $Q<P_{E}$ since $Q^{\prime} \in \mathscr{Z}$ and $Q^{\prime} \perp Q \Rightarrow Q+Q^{\prime} \in \mathscr{Z}$ by axiom $\mathbf{A 4}$. Hence $P_{E} \cong \mathbf{O}$ as required.

Weak continuity of a $\mathrm{CP} \leqq$ on $\mathscr{P}(\mathscr{H})$ immediately implies a number of useful topological properties for the $\leqq$ topology. We list some of these below:

Proposition 2.4. Let $\mathscr{H}$ be an infinite dimensional Hilbert space and $\leqq$ be a continuous $\mathrm{CP}$ on $\mathscr{P}(\mathscr{H})$. Then the following are all true.

i) $\mathscr{P}(\mathscr{H})$ is $\leqq$ connected.

ii) $\mathscr{P}(\mathscr{H})$ is order complete, i.e. every non-empty subset of $\mathscr{P}(\mathscr{H})$ has an inf and a sup with respect to $\leqq$ and hence $\mathscr{P}(\mathscr{H})$ is $\leqq$ compact.

iii) In the $\leqq$ topology, $\mathscr{P}(\mathscr{H})$ is pseudometrizable and complete. 
Proof. i) Let $J=K^{\perp}$, where $K$ is the set of all $\phi \in \mathscr{H}$ such that $P_{\phi} \cong \mathbf{O}$. Then, by Proposition 2.3 (i), $J$ is separable. Thus, if $J$ is infinite dimensional, $\mathscr{P}(J)$ is weakly and hence $\leqq$ connected. By Proposition 2.3 (ii), $P_{J} \cong \mathbf{l}_{\mathscr{H}}$, and the result follows at once for $J$ infinite dimensional. If $J$ is finite dimensional the above argument may be used with $J$ replaced by $J_{0}$, where $J_{0}$ is any separable infinite dimensional subspace of $\mathscr{H}$ containing $J$ as a subspace.

ii) This follows from (i) see, for example, [7, Chap. $1 \mathrm{~J}$ ].

iii) Let $J$ be as in (i). Then, by Proposition $2.3, \mathscr{P}(J)$ is weakly and hence $\leqq$ second countable. Hence $[\mathscr{P}(J)]$ is a regular second countable topological space under the induced $\leqq$ topology and is therefore $\leqq$ metrizable by Urysohn's metrizability theorem. The corresponding metric pseudometrizes $\mathscr{P}(J)$ (or $\left.\mathscr{P}\left(J_{0}\right)\right)$ and hence also $\mathscr{P}(\mathscr{H})$. Since $\mathscr{P}(\mathscr{H})$ is order complete it is complete as a pseudometric space.

\section{Results}

In this section, $\mathscr{H}_{0}, \psi_{0}$ and $\mathscr{H}$ are defined as follows: $\mathscr{H}_{0}$ is an infinite dimensional real or complex Hilbert space. $\psi_{0} \in \mathscr{H}_{0}$ is a fixed non-zero vector and $\mathscr{H}$ is its orthogonal complement in $\mathscr{H}_{0} . \mathscr{P}(\mathscr{H})$ is endowed with a continuous $\mathrm{CP} \leqq$. We start with a number of technical results.

Lemma 3.1. Let $x$ and $y$ be any positive reals such that $x+y \leqq 1$. Then for any $R \in \mathscr{P}_{\sigma}(\mathscr{H})$ there exist sequences $P_{j}$ and $Q_{j}$ in $\mathscr{P}_{\sigma}(\mathscr{H})$ satisfying:

i) $P_{j} \perp Q_{j} \forall j$.

ii) $P_{j} \stackrel{w}{\longrightarrow} x R$.

iii) $Q_{j} \stackrel{w}{\longrightarrow} y R$.

Further, if $R$ is infinite dimensional, then we may choose that for each $j, Q_{j} \leqq R$ and $P_{j} \leqq R$.

Proof. The case $R=\mathbf{O}$ is trivial. So assume that $R=P_{\phi}$ for some $\phi \in \mathscr{H}$. Let $\xi_{n}$ be any orthonormal sequence in the orthogonal complement of $\phi$. Clearly we can find another orthonormal sequence $\eta_{n}$, also in the orthogonal complement of $\phi$ satisfying $\left\langle\xi_{n} \mid \eta_{n}\right\rangle=\Gamma=\frac{x^{\frac{1}{2}} y^{\frac{1}{2}}}{(1-x)^{\frac{1}{2}}(1-y)^{\frac{1}{2}}} \forall n$. Note that $\Gamma \leqq 1$ for all allowed $x$ and $y$. Thus $\left\langle a \xi_{n}+x^{\frac{1}{2}} \phi \mid b \eta_{n}+y^{\frac{1}{2}} \phi\right\rangle=0 \forall n$, where $a=(1-x)^{\frac{1}{2}}$ and $b=-(1-y)^{\frac{1}{2}}$. Since $a \xi_{n}+x^{\frac{1}{2}} \phi \rightarrow x^{\frac{1}{2}} \phi$ and $b \eta_{n}+y^{\frac{1}{2}} \phi \rightarrow y^{\frac{1}{2}} \phi$ in the weak Hilbert space topology, $P_{a \xi_{n}+x^{\frac{1}{\phi}} \phi} \stackrel{w}{\longrightarrow} x P_{\phi}$ and $P_{b \xi_{n}+y^{\frac{1}{1}} \phi} \stackrel{w}{\longrightarrow} y P_{\phi}$. Hence the result is true for $R \in \mathscr{P}_{1}(\mathscr{H})$. Now let $R \in \mathscr{P}(\mathscr{H})$ be finite dimensional with $R=\sum_{j=1}^{N} P_{\phi_{j}}$, where $P_{\phi_{j}} \in \mathscr{P}_{1}(\mathscr{H}) \forall j$ and $P_{\phi_{j}} \perp P_{\phi_{k}}$ if $j \neq k$. Since we can always find $N$ mutually disjoint infinite orthonormal subsets of an infinite orthonormal set we can make use of the construction in the first part of the proof to erect, for each $P_{\phi_{j}}$, sequences $P_{j k}$ and $Q_{j k}$ such that

i) $P_{j k} \stackrel{w}{\longrightarrow} x P_{\phi_{j}}, Q_{j k} \stackrel{w}{\longrightarrow} y P_{\phi_{j}}$ as $k \rightarrow \infty$,

ii) $P_{j k} \perp Q_{j k} \forall j, k$,

iii) $P_{j k} \perp Q_{j^{\prime} k} \forall j, j^{\prime}, k$,

iv) $P_{j k} \perp P_{j^{\prime} k^{\prime}}$ and $Q_{j k} \perp Q_{j^{\prime} k^{\prime}}$ if $j \neq j^{\prime}$ or $k \neq k^{\prime}$. 
Hence $\sum_{j=1}^{N} P_{j k} \stackrel{w}{\longrightarrow} x R$ and $\sum_{j=1}^{N} Q_{j k} \stackrel{w}{\longrightarrow} y R$ as $k \rightarrow \infty$. Further, $\sum_{j=1}^{N} P_{j k} \perp \sum_{j=1}^{N} Q_{j k} \forall k$, and the result follows for $R$ finite dimensional.

Finally we consider the case where $R$ is infinite dimensional. Let $R_{n} \stackrel{w}{\longrightarrow} R$, where $R_{n}$ is finite dimensional for all $n$. From the above case we can construct, for each $n$, sequences $Q_{n j}$ and $P_{n j}$ in $\mathscr{P}_{\sigma}(\mathscr{H})$ such that $Q_{n j} \perp P_{n j} \forall j$ with $Q_{n j} \stackrel{w}{\longrightarrow} y R_{n}$ and $P_{n j} \stackrel{w}{\longrightarrow} x R_{n}$ as $j \rightarrow \infty$. Since $y R_{n} \stackrel{w}{\longrightarrow} y R$ and $x R_{n} \stackrel{w}{\longrightarrow} x R$, we can clearly find sequences from the $Q_{n j}$ and the $P_{n j}$ with required property. The last statement of the lemma follows by application of this last case to the situation where $\mathscr{H}=E$ and $R=\mathbf{l}_{E}\left(=P_{E}\right)$; completing the proof of the lemma.

Lemma 3.2. Let $P_{j}$ and $Q_{j}$ be sequences in $\mathscr{P}_{\sigma}(\mathscr{H})$ both weakly converging to one limit in $\mathscr{B}(\mathscr{H})$. Then $P_{j}$ and $Q_{j}$ both $\leqq$ converge to one limit in $\mathscr{P}(\mathscr{H})$.

Proof. There clearly exists a separable subspace $\mathscr{K}$ of $\mathscr{H}$ such that for all $j \in \mathbf{N}$, $P_{j} \in \mathscr{P}(\mathscr{K})$ and $Q_{j} \in \mathscr{P}(\mathscr{K})$. As $\mathscr{K}$ is separable the unit ball of $\mathscr{B}(\mathscr{K})$ is weakly metrizable, say with metric $d_{w}$. Let pseudometric $d_{\preceq}$ generate the $\leqq$ topology on $\mathscr{P}(\mathscr{H})$. Since $P_{j}$ and $Q_{j}$ are both $d_{w}$ Cauchy, they are also both $\overline{\bar{d}}_{\preceq}$ Cauchy. By $d_{\preceq}$ completeness there exist $P$ and $Q$ in $\mathscr{P}(\mathscr{H})$ such that $P_{j} \supseteqq P$ and $Q_{j} \stackrel{\supseteqq}{\longrightarrow} Q$. We wish to show that $P \cong Q$. If this is not the case, then $d_{\preceq}(Q, P)>0$, so that $d_{\leqq}\left(P_{j}, Q_{j}\right) \leftrightarrow 0 \Rightarrow d_{w}\left(P_{j}, Q_{j}\right) \rightarrow 0$ as $j \rightarrow \infty$ by continuity of $\leqq$. This contradicts the hypothesis of the lemma. Hence the result.

The following result shows a limited form of joint $\leqq$ continuity of orthoaddition on $\mathscr{P}(\mathscr{H})$.

Proposition 3.3. Let sequences $P_{E_{j}}$ and $P_{F_{j}}$ in $\mathscr{P}_{\sigma}(\mathscr{H})$ satisfy the following conditions:

i) $P_{E_{j}} \supseteqq P_{E} \in \mathscr{P}(\mathscr{H})$,

ii) $P_{F_{j}} \stackrel{\supseteqq}{\longrightarrow} P_{F} \in \mathscr{P}(\mathscr{H})$,

iii) $P_{E_{j}} \perp P_{F_{k}} \forall j, k \in \mathbf{N}$.

Then there exist $P_{E^{\prime}}$ and $P_{F^{\prime}}$ in $\mathscr{P}(\mathscr{H})$ such that $P_{E^{\prime}} \cong P_{E}, P_{F^{\prime}} \cong P_{F}, P_{E^{\prime}} \perp P_{F^{\prime}}$ and such that $P_{E_{j}}+P_{F_{j}} \supseteqq P_{E^{\prime}}+P_{F^{\prime}}$.

Proof. Let $\mathscr{L}$ be the minimal subspace of $\mathscr{H}$ such that $\forall j \in \mathbf{N}, P_{E_{j}} \mathscr{L}=E_{j}$ (that is $\mathscr{L}=\overline{S p \bigcup_{j \in \mathrm{N}} E_{j}}$. If $\mathscr{L}$ is infinite dimensional, then $\mathscr{P}(\mathscr{L})$ is also complete under the $\leqq$ pseudometric. Hence there exists $P_{E^{\prime}} \in \mathscr{P}(\mathscr{L})$ such that $P_{E^{\prime}} \cong P_{E}$. The same conclusion can be reached if $\mathscr{L}$ is finite dimensional by taking $P_{E^{\prime}}$ to be the norm (=weak) limit of a suitable subsequence of the $P_{E_{j}}$. Similarly let $\mathscr{M}$ be the minimal subspace of $\mathscr{H}$ such that $\forall j \in \mathbf{N}, P_{F_{j}} \mathscr{M}=F_{j}$. Again there is a $P_{F^{\prime}} \in \mathscr{P}(\mathscr{M})$ such that $P_{F^{\prime}} \cong P_{F}$. Clearly $\mathscr{M} \perp \mathscr{L}$ so that $P_{E^{\prime}} \perp P_{F^{\prime}}$. Now we show that $P_{E_{j}}+P_{F_{j}} \supseteqq P_{E^{\prime}}$ $+P_{F^{\prime}}$. We examine three cases:

Case i. $P_{E_{j}}$ and $P_{F_{j}}$ are both $\leqq$ increasing.

Let $j \geqq k$, then $P_{E_{k}}+P_{F_{k}} \leqq \bar{P}_{E_{k}}+P_{F_{j}} \leqq P_{E_{j}}+P_{F_{j}}$, where both inequalities follow from axiom A4. Hence $P_{E_{j}}+P_{F_{j}}$ is also $\supseteqq$ increasing. Thus $\left[P_{E_{j}}+P_{F_{j}}\right] \supseteqq$ $\leqq \sup _{j \in \mathbf{N}}\left(P_{E_{j}}+P_{F_{j}}\right)=\left[P_{E^{\prime}}+P_{F^{\prime}}\right]$ and the result follows. 
Case ii. $P_{E_{j}}$ and $P_{F_{j}}$ are both $\leqq$ decreasing.

From case (i) above, $\left[P_{E_{j}}+P_{F_{j}}\right] \leqq \inf _{j \in \mathbb{N}}\left(P_{E_{j}}+P_{F_{j}}\right)=\left[P_{E^{\prime}}+P_{F^{\prime}}\right]$.

Case iii. One of $P_{E_{j}}$ and $P_{F_{j}}$ is $\leqq$ increasing and the other $\leqq$ decreasing.

Assume, without losing generality, that $P_{E_{j}}$ is the $\leqq$ increasing one. Then $P_{E^{\prime}}+P_{F_{j}}$ and $P_{F^{\prime}}+P_{E_{j}}$ are $\leqq$ decreasing and $\leqq$ increasing respectively and hence both $\leqq$ converge to $P_{E^{\prime}}+P_{F^{\prime}}$. But by axiom $\mathbf{A} \overline{4}, P_{E^{\prime}}+P_{F_{j}} \leqq P_{E_{j}}+P_{F_{j}} \leqq P_{E_{j}}+P_{F^{\prime}}, \forall j$, and the result follows.

Now let $P_{E_{j}}$ and $P_{F_{j}}$ satisfy the hypothesis of the proposition but otherwise be arbitrary. Let $P_{E_{j_{k}}}+P_{F_{j_{k}}}$ be a $\leqq$ convergent subsequence of $P_{E_{j}}+P_{F_{j}}$. The subsequences $P_{E_{j_{k}}}$ and $P_{F_{j_{k}}}$ will in turn have subsequences which will fall in one of the three cases above. Thus every $\leqq$ convergent subsequence of $P_{E_{j}}+P_{F_{j}} \leqq$ converges to $P_{E^{\prime}}+P_{F^{\prime}}$ and proof of the proposition follows.

Lemma 3.4. For any $P \in \mathscr{P}_{\sigma}(\mathscr{H})$ such that $P \prec 1$, the following statements are equivalent.

i) $P \cong \mathbf{O}$.

ii) For some $x>0$, there exists a sequence $P_{j}$ in $\mathscr{P}_{\sigma}(\mathscr{H})$ such that $P_{j} \stackrel{w}{\longrightarrow} x P$ and $P_{j} \stackrel{\supseteqq}{\longrightarrow}$.

iii) For each $y \in[0,1]$ there exists a sequence $P_{j}$ in $\mathscr{P}_{\sigma}(\mathscr{H})$ such that $P_{j} \stackrel{w}{\longrightarrow} y P$ and $P_{j} \stackrel{\supseteqq}{\longrightarrow}$.

Proof. (i) $\Rightarrow$ (ii), (iii) $\Rightarrow$ (i), and (iii) $\Rightarrow$ (ii) are trivial. We prove the result by showing (ii) $\Rightarrow$ (i) and then (i) $\Rightarrow$ (iii)

(ii) $\Rightarrow$ (i):

Condition (ii) implies the following:

(a) $Q_{j} \stackrel{w}{\longrightarrow} y P$ and $y \leqq x \Rightarrow Q_{j} \stackrel{\leqq}{\longrightarrow} \mathbf{O}$.

(b) For $P$ finite dimensional, $Q_{j} \stackrel{w}{\longrightarrow}(x+y) P \Rightarrow Q_{j} \leqq \mathbf{O}$ if $0 \leqq y \leqq x$ and $x+y \leqq 1$.

Result (a) is a straightforward consequence of Lemmas 3.1 and 3.2 but (b) needs more work because joint $\leqq$ continuity of ortho-addition on $\mathscr{P}(\mathscr{H})$ has not been established. We now give a proof: By Lemma 3.1 there exist sequences $P_{E_{j}}$ and $P_{F_{j}}$ in $\mathscr{P}(\mathscr{H})$ such that $P_{E_{j}} \perp P_{F_{j}} \forall j, P_{E_{j}} \stackrel{w}{\longrightarrow} x P$ and $P_{F_{j}} \stackrel{w}{\longrightarrow} y P$. Lemma 3.2 and result (a) respectively give $P_{E_{j}} \supseteqq \mathbf{O}$ and $P_{F_{j}} \supseteqq \mathbf{O}$. Moreover, because $P$ is a finite rank projection and $P \prec \mathbf{1}$, we can arrange that $K=\left(\cup_{j} E_{j}\right)^{\perp}$ is infinite dimensional and $\mathbf{O} \prec P_{K}$. Consequently, there exists a $\leqq$ decreasing sequence $P_{\phi_{j}}$ in $\mathscr{P}_{1}(K)$ such that $\mathbf{O} \prec P_{\phi_{j}} \forall j$ and $P_{\phi_{j}} \stackrel{\supseteqq}{\longrightarrow}$. Since an appropriate subsequence of the $P_{\phi_{j}}$ can always be found, we may assume that $P_{F_{j}} \leqq P_{\phi_{j}} \forall j$. Thus by $\mathbf{A} 4$ and Lemma 3.3, $P_{E_{j}}+P_{F_{j}} \leqq P_{E_{j}}+P_{\phi_{j}} \supseteqq \mathbf{O}$, giving the result (b). Repeated application (finitely many times) of result (b) eventually leads to $P \cong \mathbf{O}$ for $P$ finite dimensional.

Now let $P$ be infinite dimensional. There exists a sequence of finite dimensional projections $P_{j}^{\prime}$, such that $P_{j}^{\prime}<P \forall j$ and, such that $P_{j}^{\prime} \stackrel{\supseteqq}{\longrightarrow} P$. It is clear that for any $t \in[0,1], P_{j} \stackrel{w}{\longrightarrow} t P$ and $P_{j} \stackrel{\supseteqq}{\longrightarrow} \mathbf{O}$ together imply that for each fixed $j$, if the sequence 
$P_{j k}^{\prime} \stackrel{w}{\longrightarrow} t P_{j}^{\prime}$ as $k \rightarrow \infty$, then $P_{j k}^{\prime} \stackrel{\preceq}{\longrightarrow} \mathbf{O}$ as $k \rightarrow \infty$. This gives $P_{j}^{\prime} \cong \mathbf{O} \forall j$. Again this leads to $P \cong \mathbf{O}$ and so wwe have (ii) $\Rightarrow$ (i).

(i) $\Rightarrow$ (iii). Let $x, y \in[0,1]$ be such that $x+y=1$. Then by Lemma 3.1 there exist sequences $S_{j}$ and $T_{j}$ in $\mathscr{P}_{\sigma}(\mathscr{H})$ such that $S_{j} \perp T_{j} \forall j$ and such that $S_{j} \stackrel{w}{\longrightarrow} x P$ and $T_{j} \stackrel{w}{\longrightarrow} y P$. Hence $S_{j}+T_{j} \stackrel{w}{\longrightarrow} P \cong \mathbf{O}$. This clearly implies that $S_{j} \stackrel{\supseteqq}{\longrightarrow}$ and $T_{j} \supseteqq \mathbf{O}$ and the result follows. Hence the lemma is proved.

Proposition 3.5. Let $R \in \mathscr{P}_{\sigma}(\mathscr{H})$ be such that $\operatorname{dim} R$ and $\operatorname{dim} R^{\perp}$ are both infinite and such that $\mathbf{O} \prec R$ and $\mathbf{O} \prec R^{\perp}$. Let $P_{j}$ and $Q_{j}$ be sequences in $\mathscr{P}_{\sigma}(\mathscr{H})$ such that $P_{j} \stackrel{w}{\longrightarrow} s R$, $Q_{j} \stackrel{w}{\longrightarrow} t R, P_{j} \supseteqq P$ and $Q_{j} \stackrel{\supseteqq}{\longrightarrow} Q$. Then $Q \leqq P \Leftrightarrow t \leqq s$.

Proof. First we note that $s, t \in[0,1]$ and that given any $x \in[0,1]$ there always exists a sequence $S_{j}$ in $\mathscr{P}_{\sigma}(\mathscr{H})$ which weakly converges to $x R$. If $s=t$ then by Lemma 3.2, $P \cong Q$. So we let $s>t$; we wish to show that $Q \prec P$. Now there exists sequences $\widetilde{Q}_{j}$ and $\widetilde{P}_{j}$ in $\mathscr{P}_{\sigma}(\mathscr{H})$ such that $\widetilde{Q}_{j} \stackrel{w}{\longrightarrow} t R, \widetilde{P}_{j} \stackrel{w}{\longrightarrow}(s-t) R, \widetilde{P}_{j} \perp \widetilde{Q}_{j} \forall j$ and such that for all $j$, $\widetilde{P}_{j}<R, \widetilde{Q}_{j}<R$. Lemma 3.2 gives $\widetilde{P}_{j}+\widetilde{Q}_{j} \supseteqq P$. Furthermore, there clearly exist $\widetilde{P} \leqq R$ and $\widetilde{Q} \leqq R$ such that $\widetilde{P}_{j} \supseteqq \widetilde{P}$ and such that $\widetilde{Q}_{j} \supseteqq \widetilde{Q}$. As $\mathbf{O}<R$ and $s-t>0$, Lemma 3.4 gives $\mathbf{O} \prec \widetilde{P}$. Because $\mathbf{O} \prec \mathbf{R}^{\perp}$ and $\operatorname{dim} R^{1}$ is infinite, there exists $S<R^{\perp}$ such that $\mathbf{O} \prec S$ and such that for all $j$ large enough, $S \leqq \widetilde{P}_{j}$. Lemmas 3.2 and 3.3 give $\widetilde{Q}_{j}+S \stackrel{ఏ}{\longrightarrow}+S$ and A4 gives $\widetilde{Q}+S \leqq P$. Hence we have $Q \cong \widetilde{Q} \prec \widetilde{Q}+S \leqq P$ as required. Since $\leqq$ is a linear preorder, the proof of the lemma is complete.

Lemma 3.6. Suppose there exists an infinite dimensional projection $R \in \mathscr{P}(\mathscr{H})$ such that $R \cong \mathbf{O}$. Let $P$ and $Q$ be any projections in $\mathscr{P}(\mathscr{H})$ such that $P \perp R$ and such that $Q<R$ with $\operatorname{dim}(R-Q)$ infinite. Suppose that the following are true for any $x \in[0,1]$ :

i) $P_{j} \stackrel{w}{\longrightarrow} x(P+Q)$ with $P_{j} \supseteqq P_{0}$.

ii) $\widetilde{P}_{j} \stackrel{w}{\longrightarrow} x P$ with $\widetilde{P}_{j} \supseteqq \widetilde{P}_{0}$.

Then $P_{0} \cong \widetilde{P}_{0}$.

Proof. Since $\operatorname{dim}(R-Q)$ is infinite (required in case $\operatorname{dim} P$ is finite) we can set up sequences $S_{j}$ in $\mathscr{P}(\mathscr{H})$ and $T_{j}$, where $T_{j} \leqq R \forall j$, such that $S_{j} \perp T_{k} \forall j, k$, and such that $S_{j} \perp T_{k} \forall j, k$, and such that $S_{j} \stackrel{w}{\longrightarrow} x P, T_{j} \stackrel{w}{\longrightarrow} x Q$. Hence $S_{j}+T_{j} \stackrel{w}{\longrightarrow} x(P+Q)$ and Lemmas 3.2 and 3.3 give the required result.

Proposition 3.7. Let $K$ be the set of all $\phi \in \mathscr{H}$ such that $P_{\phi} \cong \mathbf{O}$. Define $J$ to be $K^{\perp}$, the orthogonal complement of $K$ in $\mathscr{H}$. Let the sequences $S_{j}$ and $T_{j}$ in $\mathscr{P}_{\sigma}(\mathscr{H})$ satisfy:

i) $S_{j} \stackrel{w}{\longrightarrow} s P_{J}, S_{j} \supseteqq S$.

ii) $T_{j} \stackrel{w}{\longrightarrow} t P_{J}, T_{j} \supseteqq T$.

Then $s \leqq t \Leftrightarrow S \leqq T$.

Proof. If $t=s$ then by Lemma 3.2, $S \cong T$. So we let $t<s$ and the proposition will have been proved if we can show that $S<T$. Define $J_{0}$ to be $J$ if $J$ is infinite dimensional and otherwise to be any fixed but arbitrary infinite dimensional separable subspace of $\mathscr{H}$ containing $J$ as a subspace. Let $P_{J_{1}}$ and $P_{J_{2}}$ be both infinite dimensional projections such that $P_{J_{0}}=P_{J_{1}}+P_{J_{2}}$ and such that $\mathbf{O} \prec P_{J_{1}}, \mathbf{O} \prec P_{J_{2}}$. There exist sequences $S_{1 j}, S_{2 j}, T_{1 j}$ and $T_{2 j}$ and projections $S_{1}, S_{2}, T_{1}$ and $T_{2}$ such that 
(a) $S_{1 j} \stackrel{w}{\longrightarrow} s P_{J_{1}}$ and $S_{1 j} \stackrel{\supseteqq}{\longrightarrow} S_{1}$,

(b) $S_{2 j} \stackrel{w}{\longrightarrow} S P_{J_{2}}$ and $S_{2 j} \stackrel{\supseteqq}{\longrightarrow} S_{2}$,

(c) $T_{1 j} \stackrel{w}{\longrightarrow} t P_{J_{1}}$ and $T_{1 j} \stackrel{\lessgtr}{\longrightarrow} T_{1}$,

(d) $T_{2 j} \stackrel{w}{\longrightarrow} t P_{J_{2}}$ and $T_{2 j} \stackrel{\supseteqq}{\longrightarrow} T_{2}$.

Furthermore, since $J_{1}$ and $J_{2}$ are both infinite dimensional, we can arrange that $S_{1 j} \leqq P_{J_{1}}, T_{1 j} \leqq P_{J_{1}}, S_{2 j} \leqq P_{J_{2}} T_{2 j} \leqq P_{J_{2}}$ for all $j$ and also that $S_{1} \leqq P_{J_{1}}, S_{2} \leqq P_{J_{2}}$, $T_{1} \leqq P_{J_{1}}$ and $T_{2} \leqq P_{J_{2}}$. Now $S_{1 j}+S_{2 j} \stackrel{w}{\longrightarrow} s P_{J}$ and $T_{1 j}+T_{2 j} \stackrel{w}{\longrightarrow} t P_{J}$ so that Lemmas 3.2 and 3.6 give $S_{1 j}+S_{2 j} \stackrel{\leqq}{\rightrightarrows} S$ and $T_{1 j}+T_{2 j} \supseteqq T$. By Proposition 3.3, $T \cong T_{1}+T_{2}$ and $S \cong S_{1}+S_{2}$. Proposition 3.5 gives $T_{1} \prec S_{1}$ and $T_{2} \prec S_{2}$ so that by $\mathbf{A 4}$, $T_{1}+T_{2} \prec T_{1}+S_{2} \prec S_{1}+S_{2}$. Hence $T \prec S$. This completes the proof.

Lemma 3.8. Let $J$ be defined as in Proposition 3.7. Then for each $P \in \mathscr{P}(\mathscr{H})$ there exist a real $t \in[0,1]$ and a sequence $P_{k}$ in $\mathscr{P}(\mathscr{H})$ such that $P_{k} \stackrel{w}{\longrightarrow} t P_{J}$ and $P_{k} \stackrel{\rightrightarrows}{\longrightarrow} P$.

Proof. Define $J_{0}$ as in Proposition 3.7 but with the extra requirement that $J_{0}^{\perp}$ be infinite dimensional if $J \neq J_{0}$. Then, where appropriate, Lemma 3.6 implies that the statement of Lemma 3.8 is true if and only if the same statement is true when $J$ is replaced by $J_{0}$.

It is clear that for any $t \in[0,1]$, there is a sequence in $\mathscr{P}\left(J_{0}\right)$ weakly converging to $t P_{J_{0}}$. Moreover such a sequence is automatically $\leqq$ convergent to a (unique) $f(t) \in\left[\mathscr{P}\left(J_{0}\right)\right]$ for some $\left[\mathscr{P}\left(J_{0}\right)\right]$ valued function $f$ defined on $[0,1]$. We wish to show that $f$ is $\leqq$ continuous, where the $\leqq$ topology on $\left[\mathscr{P}\left(J_{0}\right)\right]$ is the obvious one arising from the natural ordering induced by $\leqq$. Let the metric $d_{w}^{\prime}$ generate the weak operator topology on the unit ball of $\mathscr{B}\left(\overline{\bar{J}}_{0}\right)$ and let the pseudo-metric $d_{\leqq}^{\prime}$ generate the $\leqq$ topology on $\mathscr{P}(\mathscr{H})$. We will also, in an obvious sense, regard $d_{\preceq}^{\prime}$ as a metric generating the above mentioned $\leqq$ topology on $\left[\mathscr{P}\left(J_{0}\right)\right]$. For each $s \in[0,1]$, let the sequence $Q_{j}(s)$ in $\mathscr{P}\left(J_{0}\right)$ satisfy $Q_{j}(s) \stackrel{w}{\longrightarrow} s P_{J_{0}}, Q_{j}(s) \stackrel{\supseteqq}{\longrightarrow} Q(s) \in \mathscr{P}\left(J_{0}\right)$, and we may assume that $d_{w}^{\prime}\left(Q_{j}(s), s P_{J_{0}}\right)<1 / j$ for all $j$ by taking a suitable subsequence. For any $\varepsilon>0$ there exists $\delta>0$ such that $|s-t|<\delta$ implies that $d_{w}^{\prime}\left(s P_{J_{0}}, t P_{J_{0}}\right)<\varepsilon / 2$. Choosing $j_{0}$ such $j_{0} \geqq 4 / \varepsilon$, we have $d_{w}^{\prime}\left(Q_{j}(s), Q_{j}(t)\right)<\varepsilon$ whenever $|s-t|<\delta$ and $j \geqq j_{0}$. Hence it follows from the weak continuity of $\leqq$ that for any $\varepsilon>0$, there exist $\delta^{\prime}>0$ and $j_{0}^{\prime}$ such that $d_{\leqq}^{\prime}\left(Q_{j}(s), Q_{j}(t)\right)<\varepsilon$ whenever $|s-t|<\delta^{\prime}$ and $j \geqq j_{0}^{\prime}$. Now suppose the sequence $t_{k} \rightarrow t$ on $[0,1]$. Then there exists $k_{0}$ such that $\left|t_{k}-t\right|<\delta^{\prime}$ for $k \geqq k_{0}$, and hence we have $d_{\geqq}^{\prime}\left(Q_{j}\left(t_{k}\right), Q_{j}(t)\right)<\varepsilon$ whenever $k \geqq k_{0}$ and $j \geqq j_{0}^{\prime}$. Since $d_{\unlhd}^{\prime}\left(Q_{j}\left(t_{k}\right), Q\left(t_{k}\right)\right) \rightarrow 0$ and $d_{\unlhd}^{\prime}\left(Q_{j}(t), Q(t)\right) \rightarrow 0 \quad$ as $j \rightarrow \infty$, we have $d_{\leqq}\left(f\left(t_{k}\right), f(t)\right)=d_{\leqq}\left(Q\left(t_{k}\right), Q(t)\right)<\varepsilon$ whenever $k \geqq k_{0}$. This gives the desired continuity of $f$. The continuity of $f$ implies that the range of $f$ is $\leqq$ connected and is a $\leqq$ interval. Since $f(0)=[\mathbf{O}]$ and $f(1)=\left[P_{J_{0}}\right]$ and since $P_{J_{0}} \cong \mathrm{I}_{\mathscr{H}}$, the lemma is proved.

We remark that by Proposition 3.7, the function $f$ defined in the above lemma is strictly $\leqq$ increasing and hence injective. We are therefore able to make the following definition:

Definition 3.9. Let $J$ be defined as in Proposition 3.7. Define the real $\leqq$ continuous function $\mu: \mathscr{P}(\mathscr{H}) \rightarrow[0,1]$ as follows:

$\mu(P)=t$ if an only if there exists a sequence $P_{k}$ in $\mathscr{P}(\mathscr{H})$ such that $P_{k} \stackrel{w}{\longrightarrow} t P_{J}$ and $P_{k} \stackrel{\leqq}{\longrightarrow}$. 
We now wish to extend the preorder $\leqq$ to all of $\mathscr{P}\left(\mathscr{H}_{0}\right)$. The extend preorder will not necessarily satisfy all of the axioms of a CP and, in particular, axiom A4 will be replaced by a weaker one. First, we prove a lemma.

Lemma 3.10. Let $[P]=\leqq \sup \mathscr{P}_{1}(\mathscr{H})$. Then $[P] \cap \mathscr{P}_{1}(\mathscr{H})$ is not empty.

Proof. Let $P_{\phi_{j}} \supseteqq P \in \leqq \sup \mathscr{P}_{1}(\mathscr{H})$, and let $P_{\phi_{j_{k}}}$ be a weakly convergent subsequence of $P_{\phi_{j}}$. Then, since the range of the weak limit of $P_{\phi_{j}}$ is one dimensional, $P_{\phi_{l k}} \stackrel{w}{\longrightarrow} t P_{\phi_{0}}$ for some $\phi_{0} \in \mathscr{H}$, where $0<t \leqq 1$. By Corollary 3.6, $t=1$ and the proof is complete.

Let $P_{F} \in \mathscr{P}\left(\mathscr{H}_{0}\right) \backslash \mathscr{P}(\mathscr{H})$, then $P_{F} \psi_{0} \neq 0$. Define $\psi_{F}$ to be $P_{F} \psi_{0} /\left\|P_{F} \psi_{0}\right\|$, then $\psi_{F}$ may be written as $a \phi_{0}+b \psi_{0}+c \xi$, where $\xi \perp \psi_{0}, \xi \perp \phi_{0}$ and $\phi_{0}$ is a fixed vector in $\mathscr{H}$ such that $P_{\phi_{0}}$ is $\leqq$-maximal in $\mathscr{P}_{1}(\mathscr{H})$ (Lemma 3.10). Accordingly, any $P_{F} \in \mathscr{P}\left(\mathscr{H}_{0}\right) \backslash \mathscr{P}(\mathscr{H})$ may be "canonically" decomposed, with respect to the pair $\left(\phi_{0}, \psi_{0}\right)$, as follows:

$$
P_{F}=P_{E}+P_{\psi_{F}}=P_{E}+P_{a \phi_{0}+b \psi_{0}+c \xi},
$$

where $b \neq 0, P_{E} \in \mathscr{P}(\mathscr{H}), \xi \perp \psi_{0}, \xi \perp \phi_{0}$. Moreover, the decomposition is essentially unique, that is:

$P_{E}+P_{a \phi_{0}+b \psi_{0}+c \xi}=P_{E^{\prime}}+P_{a^{\prime} \phi_{0}+b^{\prime} \psi_{0}+c^{\prime} \xi^{\prime}}$ are canonical decompositions implies that $E=E^{\prime}, a=a^{\prime}, b=b^{\prime}$, and $c \xi=c^{\prime} \xi^{\prime}$.

Lemma 3.11. Let $P_{F_{j}}$ be a net in $\mathscr{P}\left(\mathscr{H}_{0}\right)$ weakly converging to $P_{F} \in \mathscr{P}\left(\mathscr{H}_{0}\right)$. If $P_{E_{j}}+P_{a, \phi_{0}+b_{j} \psi_{0}+c_{j} \xi_{j}}$ and $P_{E}+P_{a \phi_{0}+b \psi_{0}+c \xi}$ are the canonical decompositions of $P_{F_{j}}$ and $P_{F}$ respectively then

i) $P_{E_{j}} \stackrel{w}{\longrightarrow} P_{E}$ and

ii) $P_{a_{j} \phi_{0}+b_{j} \psi_{0}+c_{j} \xi_{j}} \stackrel{w}{\longrightarrow} P_{a \phi_{0}+b \psi_{0}+c \xi}$

Proof. Since we can always find appropriate subnets, we assume, without loss of generality, that $P_{E_{j}} \stackrel{w}{\longrightarrow} A \in \mathscr{B}^{+}(\mathscr{H})$ and $P_{a_{j} \phi_{0}+b_{j} \psi_{0}+c_{j} \xi_{j}} \stackrel{w}{\longrightarrow} k P_{\phi}$, for some positive $k$ and some $\phi \in \mathscr{H}_{0}$. Thus we have

$$
P_{E}+P_{a \phi_{0}+b \psi_{0}+c \xi}=A+k P_{\phi} \Rightarrow P_{a \phi_{0}+b \psi_{0}+c \xi} \psi_{0}=k P_{\phi} \psi_{0} \Rightarrow \phi=m\left(a \phi_{0}+b \psi_{0}+c \xi\right)
$$

for some $m$ such that $|m|=1$. This in turn implies that $k=1$, leading to the desired result.

Suppose that $a, b \in \mathbf{C}$ with $b \neq 0$. One easily shows that there exists a unique $t \geqq 0$ such that $|a|^{2}+|b|^{2}=|a+t b|^{2}$. Furthermore, $t$ depends continuously on $a$ and $b$. Now we define the function $\tilde{\mu}: \mathscr{P}\left(\mathscr{H}_{0}\right) \rightarrow \mathbf{R}$ as follows:

where

$$
\begin{gathered}
\tilde{\mu}\left(P_{E}\right)=\mu\left(P_{E}\right) \quad \text { if } \quad P_{E} \in \mathscr{P}(\mathscr{H}) . \\
\tilde{\mu}\left(P_{E}+P_{a \phi_{0}+b \psi_{0}+c \xi}\right)=\mu\left(P_{E}\right)+\mu\left(P_{(a+t b) \phi_{0}+c \xi}\right),
\end{gathered}
$$

$$
P_{E}+P_{a \phi_{0}+b \psi_{0}+c \xi} \in \mathscr{P}\left(\mathscr{H}_{0}\right) \backslash \mathscr{P}(\mathscr{H})
$$

is in canonical decomposition and where $|a|^{2}+|b|^{2}=|a+t b|^{2}, t \geqq 0$.

Lemma 3.12. $\tilde{\mu}$ satisfies the following:

(a) $\tilde{\mu}$ is weakly continuous on $\mathscr{P}\left(\mathscr{H}_{0}\right) \backslash \mathscr{P}(\mathscr{H})$.

(b) If $P_{\phi} \in \mathscr{P}_{1}(\mathscr{H})$ then there exists a sequence $P_{\phi_{j}}$ in $\mathscr{P}\left(\mathscr{H}{ }_{0}\right) \backslash \mathscr{P}(\mathscr{H})$ such that $P_{\phi_{j}} \stackrel{w}{\longrightarrow} P_{\phi}$ and such that $\tilde{\mu}\left(P_{\phi_{j}}\right) \rightarrow \tilde{\mu}\left(P_{\phi}\right)$. 
Proof. Let $P_{F_{j}} \stackrel{w}{\longrightarrow} P_{F} \in \mathscr{P}\left(\mathscr{H}_{0}\right) \backslash \mathscr{P}(\mathscr{H})$ and let $P_{E_{j}}+P_{a_{j} \phi_{0}+b_{j} \psi_{0}+c_{j} \xi_{j}}$ and $P_{E}+P_{a \phi_{0}+b \psi_{0}+c \xi}$ be the canonical decomposition of $P_{F_{j}}$ and $P_{F}$ respectively. As $b \neq 0$, Lemma 3.11 implies that $P_{E_{j}}+P_{\left(a_{j}+t_{j} b_{j}\right) \phi_{0}+c_{j} \xi_{j}} \stackrel{w}{\longrightarrow} P_{E}+P_{(a+t b) \phi_{0}+c \xi}$, where $t$ and $t_{j}$ have obvious meaning. The weak continuity of $\mu$ gives $\tilde{\mu}\left(P_{F_{j}}\right) \rightarrow \tilde{\mu}\left(P_{F}\right)$ as required. This proves part (a).

To prove part (b) let $P_{a_{j} \phi_{0}+b_{j} \psi_{0}+c_{j} \xi_{j}}$ and $P_{a \phi_{0}+c \xi}$ be the "canonical forms" of $P_{\phi_{j}}$ and $P_{\phi}$ respectively. Explicit calculation shows that the choice $c_{j} \xi_{j}=c \xi, b_{j}=a \sqrt{1 / j}$ and $a_{j}=a \sqrt{1-1 / j} \forall j$, ensures the desired convergence for the case where $a \neq 0$. If $a=0$ we make the choice $a_{j}=0, \xi_{j}=\xi, c_{j}=c \sqrt{1-1 / j}$ and $b_{j}=c \sqrt{1 / j} \forall j$.

Define the preorder $\unlhd$ on $\mathscr{P}\left(\mathscr{H}_{0}\right)$ as follows:

$$
P \unlhd Q \Leftrightarrow \tilde{\mu}(P) \leqq \tilde{\mu}(Q) .
$$

$\unlhd$ is clearly an ECP on $\mathscr{P}\left(\mathscr{H}_{0}\right)$ and as such, an extension of $\leqq$. It also satisfies (cf. axiom A4):

A'4. Let $P_{E}, P_{F} \in \mathscr{P}(\mathscr{H})$ and let $P_{\phi}, P_{\psi} \in \mathscr{P}_{1}\left(\mathscr{H}_{0}\right)$.

(a) If $P_{E} \perp P_{\phi}$ and $P_{E} \perp P_{\psi}$ then $P_{\psi} \unlhd P_{\phi} \Leftrightarrow P_{E}+P_{\psi} \unlhd P_{E}+P_{\phi}$.

(b) If $P_{E} \perp P_{\phi}$ and $P_{F} \perp P_{\phi}$ then $P_{F} \unlhd P_{E} \Leftrightarrow P_{F}+P_{\phi} \unlhd P_{E}+P_{\phi}$.

Lemma 3.13. Let $P_{E}, P_{F} \in \mathscr{P}(\mathscr{H})$ be finite dimensional and let $P_{\phi}, P_{\psi} \in \mathscr{P}_{1}(\mathscr{H})$ be such that

i) $P_{E} \perp P_{\phi}, P_{F} \perp P_{\psi}$

ii) $P_{F} \leqq P_{E}, P_{\psi} \leqq P_{\phi}$.

Then $P_{F}+P_{\psi} \leqq P_{E}+P_{\phi}$.

Proof. If $P_{\psi} \cong \mathbf{O}$ then the result is clearly true. Now let $\mathbf{O} \prec P_{\psi}$. There exists $P_{\xi} \in \mathscr{P}_{1}(\mathscr{H})$ such that $P_{\xi} \perp P_{F}, P_{\xi} \perp P_{E}$ and $P_{\xi} \leqq P_{\psi}$. Using the continuity properties of Lemma 3.12 we can find $P_{\chi} \in \mathscr{P}_{1}\left(\mathscr{H}_{0}\right)$, where $\chi$ is a linear combination of $\xi$ and $\psi_{0}$ and satisfies $P_{\psi} \unlhd P_{\chi} \unlhd P_{\phi}$. By axiom $\mathbf{A}^{\prime} 4$ we have: $P_{F}+P_{\psi} \unlhd P_{F}+P_{\chi} \unlhd P_{E}$ $+P_{\chi} \unlhd P_{E}+P_{\phi}$. Hence the result.

Proposition 3.14. Let $P_{E} \in \mathscr{P}(\mathscr{H})$ be finite dimensional and let $P_{\phi} \in \mathscr{P}_{1}(\mathscr{H})$ be such that $P_{E} \perp P_{\phi}$. Let sequences $P_{E_{j}}$ and $P_{\phi_{j}}$ in $\mathscr{P}(\mathscr{H})$ and in $\mathscr{P}_{1}(\mathscr{H})$ respectively be both $\supseteqq$ increasing with $P_{E_{j}}$ finite dimensional for all j. Suppose $P_{E_{j}} \stackrel{\supseteqq}{\longrightarrow} P_{E}$ and $P_{\phi_{j}} \stackrel{\supseteqq}{\longrightarrow} P_{\phi}$ with $P_{E_{j}} \perp P_{\phi_{j}} \forall j$, then $P_{E_{j}}+P_{\phi_{j}} \supseteqq P_{E}+P_{\phi}$.

Proof. By Lemma 3.13, $P_{E_{j}}+P_{\phi_{j}}$ is $\leqq$ increasing, hence $\leqq$ convergent. $P_{E}$ is finite dimensional, hence there exist strictly $\leqq$ increasing sequences $P_{\tilde{E}_{j}}$ and $P_{\tilde{\phi}_{j}}$ in $\mathscr{P}(\mathscr{H})$ and $\mathscr{P}_{1}(\mathscr{H})$ respectively such that $P_{\tilde{E}_{j}}$ is finite dimensional for all $j$, $P_{\tilde{E}_{j}} \stackrel{\supseteqq}{\longrightarrow} P_{E}, P_{\tilde{\phi}_{j}} \stackrel{\supseteqq}{\longrightarrow} P_{\phi}$ and such that $P_{\tilde{E}_{j}} \perp P_{\tilde{\phi}_{k}}$ for all $j$ and $k$. By Proposition 3.3, $P_{\tilde{E}_{j}}+P_{\tilde{\phi}_{j}} \supseteqq P_{E}+P_{\phi}$; moreover $P_{\tilde{E}_{j}}+P_{\tilde{\phi}_{j}}$ is strictly $\leqq$ increasing. Clearly the sequences $P_{\tilde{E}_{j}}+P_{\tilde{\phi}_{j}}$ and $P_{E_{j}}+P_{\phi_{j}}$ converge to the same limit and the result follows.

Proposition 3.15. Let $P_{\phi_{j}} \in \mathscr{P}_{1}(\mathscr{H}): 1 \leqq j \leqq N<\infty$ be such that $P_{\phi_{j}} \perp P_{\phi_{k}}$ if $j \neq k$. Then

$$
\mu\left(\sum_{j=1}^{N} P_{\phi_{j}}\right)=\sum_{j=1}^{N} \mu\left(P_{\phi_{j}}\right) .
$$


Proof. Suppose that the result is true for some $N \geqq 1$. Let $P_{\psi_{j}}: 1 \leqq j \leqq N+1$ satisfy the hypothesis of the proposition. Let $P_{E}=\sum_{j=1}^{N} P_{\psi_{j}}, \mu\left(P_{E}\right)=t$ and $\mu\left(P_{\psi_{N+1}}\right)=s$, then $\mu\left(P_{E}\right)=\sum_{j=1}^{N} \mu\left(P_{\phi_{j}}\right)$. Clearly we can find $\leqq$ increasing sequences $P_{E_{j}}$ and $P_{\phi_{j}}$, with the $P_{E_{j}}$ finite dimensional, such that

(i) $P_{E_{j}} \stackrel{w}{\longrightarrow} t P_{J}, P_{\phi_{j}} \stackrel{w}{\longrightarrow} s P_{J} \quad$ ( $J$ as defined in Proposition 3.7)

(ii) $P_{E_{j}} \stackrel{\supseteqq}{\longrightarrow} P_{E}, P_{\phi_{j}} \supseteqq P_{\psi_{N+1}}$ and

(iii) $P_{E_{j}} \perp P_{\phi_{j}} \forall j$. Hence

By Proposition 3.14, $P_{E_{j}}+P_{\phi_{j}} \supseteqq P_{E}+P_{\psi_{N+1}}$, so that $\mu\left(P_{E}+P_{\psi_{N+1}}\right)=s+t$.

$$
\mu\left(\sum_{j=1}^{N+1} P_{\psi_{j}}\right)=\mu\left(P_{\psi_{N+1}}\right)+\mu\left(\sum_{j=1}^{N} P_{\psi_{j}}\right)=\sum_{j=1}^{N+1} \mu\left(P_{\psi_{j}}\right)
$$

and the result is true for $N+1$. But it is trivially true for $N=1$ and the proof is complete.

Corollary 3.16. Let the sequence $P_{\phi_{j}}$ in $\mathscr{P}_{1}(\mathscr{H})$ be such that $P_{\phi_{j}} \perp P_{\phi_{k}}$ if $j \neq k$. Then

$$
\mu\left(\sum_{j \in \mathbf{N}} P_{\phi_{j}}\right)=\sum_{j \in \mathbf{N}} \mu\left(P_{\phi_{j}}\right) \text {. }
$$

Proof. Define $P_{E_{n}}=\sum_{j=1}^{n} P_{\phi_{j}}$. Then $\forall n, \sum_{j=1}^{n} \mu\left(P_{\phi_{j}}\right)=\mu\left(P_{E_{n}}\right) \leqq \mu\left(\sum_{j=1}^{\infty} P_{\phi_{j}}\right)$. Hence the sum $\sum_{j=1}^{\infty} \mu\left(P_{\phi_{j}}\right)$ is absolutely convergent. Since $\mu$ is $\leqq$ continuous and $P_{E_{n}} \supseteqq \sum_{j=1}^{\infty} P_{\phi_{j}}$, the result follows.

\section{The Main Result}

Corollary 3.16 together with Proposition 2.3 imply that $\mu$ is in fact a completely additive Gleason measure on $\mathscr{P}(\mathscr{H})$. Hence we have the main result:

Theorem 4.1. Let $\mathscr{H}$ be an infinite dimensional (not necessarily separable) Hilbert space and $\leqq a \mathrm{CP}$ on $\mathscr{P}(\mathscr{H})$. Then the following statements are equivalent:

i) $\leqq$ can be implemented by a normal state.

ii) $\leqq$ is weakly continuous.

This result shows that, subject to the usual condition that quantum expectation values should respect the weak operator topology on the appropriate algebra of observables, there is nothing gained by departing from the traditional formulation in which expectation values are represented by normal states. Atomic Energy Agency, UNESCO and the Swedish Development Cooperation for their support and hospitality at the International Centre for Theoretical Physics, Trieste, Italy. 


\section{References}

1. Gleason, A.M.: Measures on the closed subspace of a Hilbert space. J. Math. Mech. 6, 885-893 (1957)

2. Kalmbach, G.: Measures and Hilbert lattices. Singapore: World Scientific 1986

3. Ochs, W.: Gleason measures and quantum comparative probability. Quantum Probability and Applications II (Heidelberg Proceedings), pp. 388-396. Berlin, Heidelberg, New York: Springer 1985

4. Bratteli, O., Robinson, D.W.: Operator algebras and quantum statistical mechanics. I. Berlin, Heidelberg, New York: Springer 1979

5. Gaal, S.A.: Point set topology. London, New York: Academic Press 1964

6. Reed, M., Simon, B.: Methods of modern mathematical physics, Vol. 1. London, New York: Academic Press 1980

7. Kelly, J.L.: General topology. Amsterdam: Van Nostrand 1955

Communicated by H. Araki

Received August 19, 1989; in revised form Februar 27, 1990 